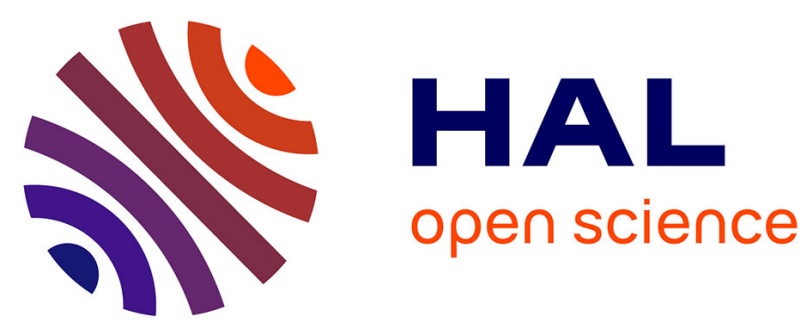

\title{
Mechanical alterations of the bone-cartilage unit in a rabbit model of early osteoarthrosis
}

\author{
Sarah Pragnère, C. Boulocher, Ophélie Pollet, Catherine Bosser, Aurélie \\ Levillain, Magali Cruel, Thierry Hoc
}

\section{> To cite this version:}

Sarah Pragnère, C. Boulocher, Ophélie Pollet, Catherine Bosser, Aurélie Levillain, et al.. Mechanical alterations of the bone-cartilage unit in a rabbit model of early osteoarthrosis. Journal of the mechanical behavior of biomedical materials, 2018, 83, pp.1-8. 10.1016/j.jmbbm.2018.03.033 . hal-02083241

\section{HAL Id: hal-02083241 \\ https://hal.science/hal-02083241}

Submitted on 28 Mar 2019

HAL is a multi-disciplinary open access archive for the deposit and dissemination of scientific research documents, whether they are published or not. The documents may come from teaching and research institutions in France or abroad, or from public or private research centers.
L'archive ouverte pluridisciplinaire HAL, est destinée au dépôt et à la diffusion de documents scientifiques de niveau recherche, publiés ou non, émanant des établissements d'enseignement et de recherche français ou étrangers, des laboratoires publics ou privés. 
Mechanical alterations of the bone-cartilage unit in a rabbit model of early osteoarthrosis

Sarah Pragnère ${ }^{1}$, Caroline Boulocher ${ }^{2}$, Ophélie Pollet ${ }^{1}$, Catherine Bosser ${ }^{1}$, Aurélie Levillain ${ }^{1}$, Magali Cruel ${ }^{1}$, Thierry Hoc ${ }^{1}$.

${ }^{1}$ LTDS UMR 5513, Ecole Centrale de Lyon, 36 avenue Guy de Collongue, 69134 Ecully

Cedex, France

${ }^{2}$ VetAgro Sup, 1 Avenue Bourgelat, 69280 Marcy-l'Étoile, France

CORRESPONDING AUHOR :

Name : Hoc Thierry

Address : LTDS Batiment G8, Ecole Centrale de Lyon, 36 Av Guy de Collongue, 69134

Ecully Cedex France

Email : Thierry.hoc@ec-lyon.fr

Tel : +33472186214 


\section{$\underline{\text { ABSTRACT }}$}

Objective: The purpose of this study was to assess mechanical properties along with microstructural modifications of the hyaline cartilage (HC), calcified cartilage (CC) and cortical plate (Ct.Pt), in an anterior cruciate ligament transection (ACLT) model.

Medial femoral condyles of six healthy rabbits (control group) and of six ACLT rabbits 6 weeks after OA induction were explanted. The zone of interest (ZOI) for all experiments was defined as the weight bearing areas of the samples. Biomechanical properties were measured using nanoindentation and morphological changes were evaluated using biphotonic confocal microscopy (BCM).

Results: All rabbits of the ACLT group displayed early PTOA. The results indicate an overall decrease in the mechanical properties of the HC, CC and Ct.Pt in the ACLT group. The average equilibrium modulus and elastic fraction of the $\mathrm{HC}$ decreased by $42 \%$ and $35 \%$, respectively, compared with control group. The elastic moduli of the CC and Ct.Pt decreased by $37 \%$ and $16 \%$, respectively, compared with control group. A stiffness gradient between CC and Ct.Pt appeared in the ACLT group. The irregularity of the cement line, quantified by its tortuosity in BCM images, was accentuated in the ACLT group compared with the control group.

Conclusions: In the ACLT model, weight-bearing stress was modified in the ZOI. This disruption of the stress pattern induced alterations of the tissues composing the bone-cartilage unit. In term of mechanical properties, all tissues exhibited changes. The most affected tissue was the most superficial: hyaline cartilage displayed the strongest relative decrease $(42 \%)$ 
followed by calcified cartilage (37\%) and cortical plate was slightly modified (16\%). This supports the hypotheses that PTOA initiates in the hyaline cartilage.

Keywords: Cartilage mechanics; Bone mechanics; Osteoarthritis; Biphoton imaging; Mineralization; Nanoindentation; Hyaline cartilage; Calcified cartilage; Subchondral bone 
Introduction

Osteoarthritis (OA) is involved in the degradation of the whole joint, including articular cartilage and subchondral bone, composing together the bone-cartilage unit $(\mathrm{BCU})(1)$. Articular cartilage is a complex viscous-elastic tissue made of several layers, tethered to the subchondral bone by the osteochondral junction. This osteochondral junction lies beneath the tidemark, which is a line that delineates the bottom of the hyaline articular cartilage i.e between the uncalcified (hyaline) and calcified cartilage. The osteochondral junction includes the mineralised tissues, i.e calcified cartilage (CC) and the subchondral cortical plate (Ct.Pt), which are separated from each other by the cement line (2). These two mineralized tissues are tightly anchored through a highly interdigitated interface and are linked by some collagen fibers that cross the cement line (3). Whether the changes in the osteochondral junction occurring in OA are a cause or a consequence of hyaline cartilage damage is still debated (4).

Mechanical modification of loads by increased focal stress or excess overall load are factors that lead to OA (5). Because one of the major roles of both the articular cartilage and osteochondral junction is to absorb and transmit forces across the whole joint, any modification of these tightly linked tissues plays an important role in OA onset and progression (6). Calcified cartilage acts as an interface between the compliant hyaline cartilage and the stiffer cortical plate, which permits load transfer while preventing large cartilage deformation (7). In particular, it is thought to transform shear stress into compression $(8,9)$. In addition, its low permeability limits the diffusion of molecules from bone towards the hyaline cartilage and therefore represents a barrier for hyaline cartilage mineralization (10). Only a few studies have focused on the mechanical properties of each individual component of the bone-cartilage unit $(11,12)$. Besides, to our knowledge, no studies have assessed the alterations of the mechanical properties of hyaline cartilage along with the osteochondral junction in a rabbit model of OA. 
Recently, several studies have focused on changes in the subchondral trabecular bone in both human (13-15) and animal models (16-18) and have shown that cortical bone plate remodelling increases during OA establishment (19). In the very early stages of OA, cortical plate thickness decreases. In later stages of OA, sclerosis is observed and the subchondral bone mineral content diminishes, which results in a weakening of the mechanical properties at the tissue scale (20). However, the evolution of calcified cartilage thickness remains unclear, especially in early OA (4). Indeed, calcified cartilage thickness evolution depends both on its progression into hyaline articular cartilage and on the progression of bone into the calcified cartilage. Furthermore, in the calcified cartilage, increased vascular invasion due to angiogenic activity has been observed in all stages of OA $(4,21,22)$. Nonetheless, osteochondral junction alterations during OA remains understudied from a mechanical point of view.

Animal models provide useful information to study the mechanisms involved in OA. Notably, they allow temporal monitoring of OA changes in the early stages. Especially, OA can be induced surgically, which mimics post traumatic OA (PTOA) that occurs in humans and results in altered load bearing $(23,24)$. In the present study, a model of anterior cruciate ligament transection (ACLT) in rabbit knees was chosen because it is a well-established model of PTOA $(19,21,25-27)$ that results in true instability-induced OA lesions (28-30). These instabilities are assumed to increase the risk of developing OA in human patients $(31,32)$, therefore understanding the course of PTOA is a major health issue.

The aim of the present study was to assess the mechanical properties of hyaline cartilage, calcified cartilage and cortical plate in an ACLT model. These mechanical modifications were correlated to changes in the morphology of the osteochondral junction using biphotonic confocal microscopy. 


\section{Materials and Methods}

\subsection{Animal model}

All of the experiments and procedures involving animals were approved by the local Ethics Committee (ComEth Anses/ENVA/UPEC number 16) and were performed in full accordance with European legislation. Twelve healthy adult male New Zealand White rabbits (six months of age, $3.8 \mathrm{~kg}$ in weight on average) free of degenerative joint disease were obtained from a licensed vendor (EUROLAP, Gosné, France). After two weeks in acclimatization and quarantine, two groups were constituted randomly: a control group (healthy, not operated) $(n=6)$ and an operated group $(n=6)$. In the operated group, experimental OA was surgically induced by ACLT and performed by a trained veterinary surgeon in the right knee (stifle) of the six rabbits. The operated limb was not immobilized postoperatively, and the rabbits were allowed to move freely in their individual cages immediately after surgery. The six rabbits of the control (healthy) group were left unoperated. After a six-week observation period, the rabbits from the control and ACLT groups were euthanized. Both knees were explanted from each animal, carefully dissected, and the menisci were detached. Meniscus and cartilage degradations, as well as osteophyte production, were graded in right knees (healthy control (n $=6)$, and operated $(n=6)$ ) using a macroscopic grading system developed by Laverty et al. (25).

\subsection{Hyaline cartilage mechanical testing}

Immediately after removal, right femurs from both control $(n=6)$ and ACLT $(n=6)$ groups were sawed above the distal epiphysis of femur and stored at $-20^{\circ} \mathrm{C}$ until they were used in experiments. The femoral condyles were thawed at room temperature for a couple of hours and then sawed along the longitudinal axis of the femur to separate the medial femoral condyles (MFC) from their lateral counterpart. Indentation-relaxation tests were performed 
following previously published protocol (33) on each sample surrounded by a physiological saline solution at ambient temperature using a commercial nanoindenter (Agilent Nanoindenter G200, ScienTec, Les Ulis, France). The indenter was a spherical sapphire tip with a radius of curvature of $0.479 \mathrm{~mm}$. The subchondral part of the condyle was glued onto a support. The zone of interest (ZOI) was defined at the load bearing area of the femoral medial condyles as previously described by Vaseenon et al (34) (figure 1-B). Indentation tests were then conducted on 3 locations in the direction of physiological loading and repeated three times. As classically performed in nanoindentation, a rubber reference material was indented before each series of tests to calibrate the device. A constant displacement rate of $5 \mu \mathrm{m} . \mathrm{s}^{-1}$ and a penetration of $40 \mu \mathrm{m}$ were imposed. The indenter displacement was then maintained for $400 \mathrm{~s}$ until equilibrium was reached. Unloading was carried out at $0.05 \mu \mathrm{m} . \mathrm{s}^{-1}$.
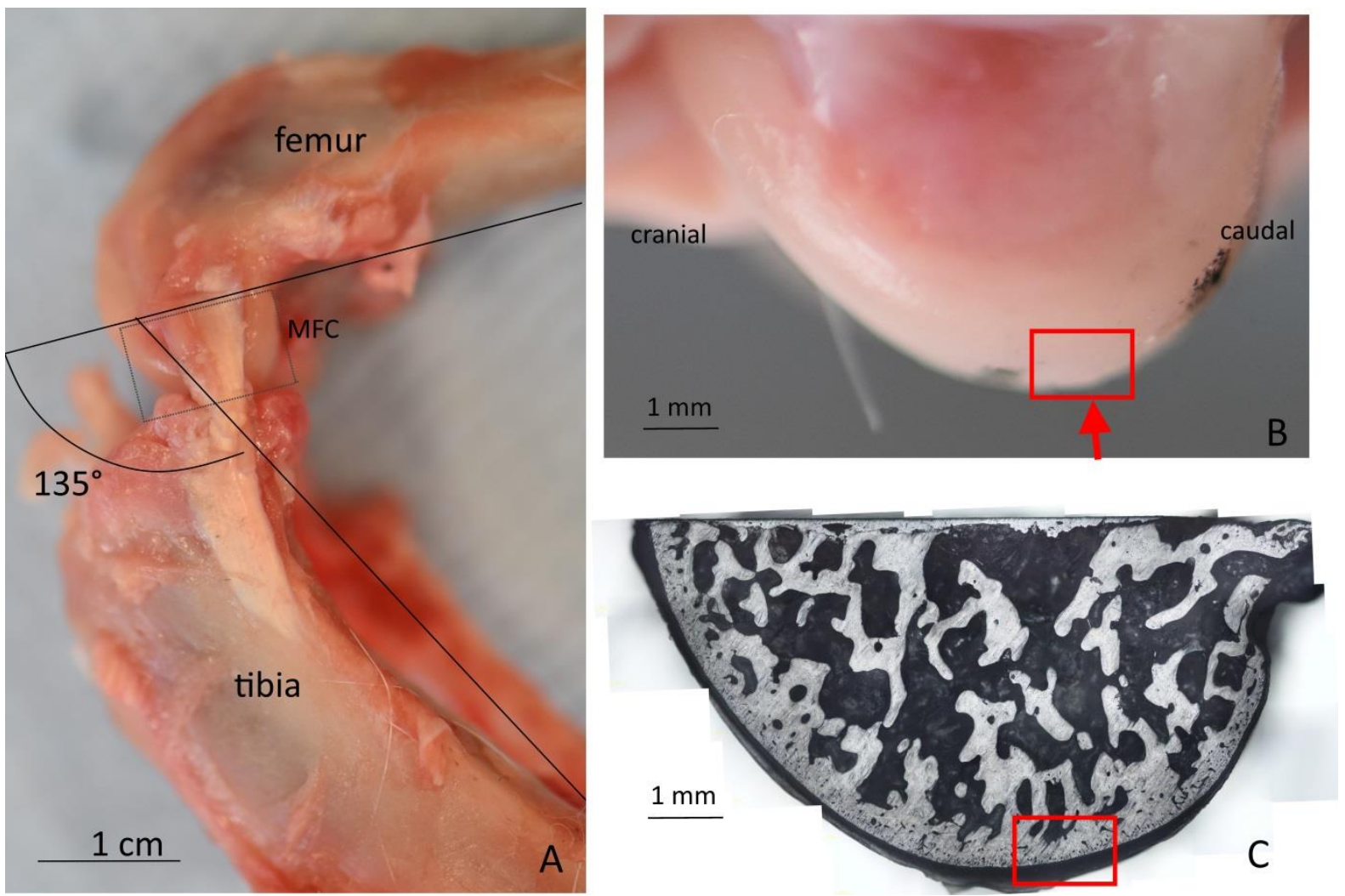

Figure 1 : Localization of the contact area between the femoral and tibial articular cartilage surfaces to define the ZOI according to Vaseenon et al. (34). A: Measurement of $135^{\circ}$ of flexion between the femur and the tibia (right limb). Both menisci were removed. B: Zoomed in image of the medial femoral condyle (MFC). The ZOI is located between the black spots (red rectangle). The red arrow indicates the indentation axe of the hyaline cartilage. $C$ : Optical microscopy of the medial condyle in the sagittal plane. The red rectangle corresponds to the ZOI. 
Instantaneous $\left(\mathrm{E}_{\mathrm{ins}}\right)$, equilibrium $\left(\mathrm{E}_{\mathrm{eq}}\right)$ elastic moduli and elastic fraction $\left(\mathrm{f}=\mathrm{E}_{\mathrm{eq}} / \mathrm{E}_{\text {ins }}\right)$ were then determined from the resultant force-time data using the viscoelastic model previously published by Levillain et al (33) with four branches. The elastic fraction describes the elastic/viscous behavior of the material ( $\mathrm{f}=1$ corresponds to a perfectly elastic material, whereas $\mathrm{f}=0$ corresponds to a perfectly viscous material). The fit of the experimental data obtained with this model is displayed in figure 2.

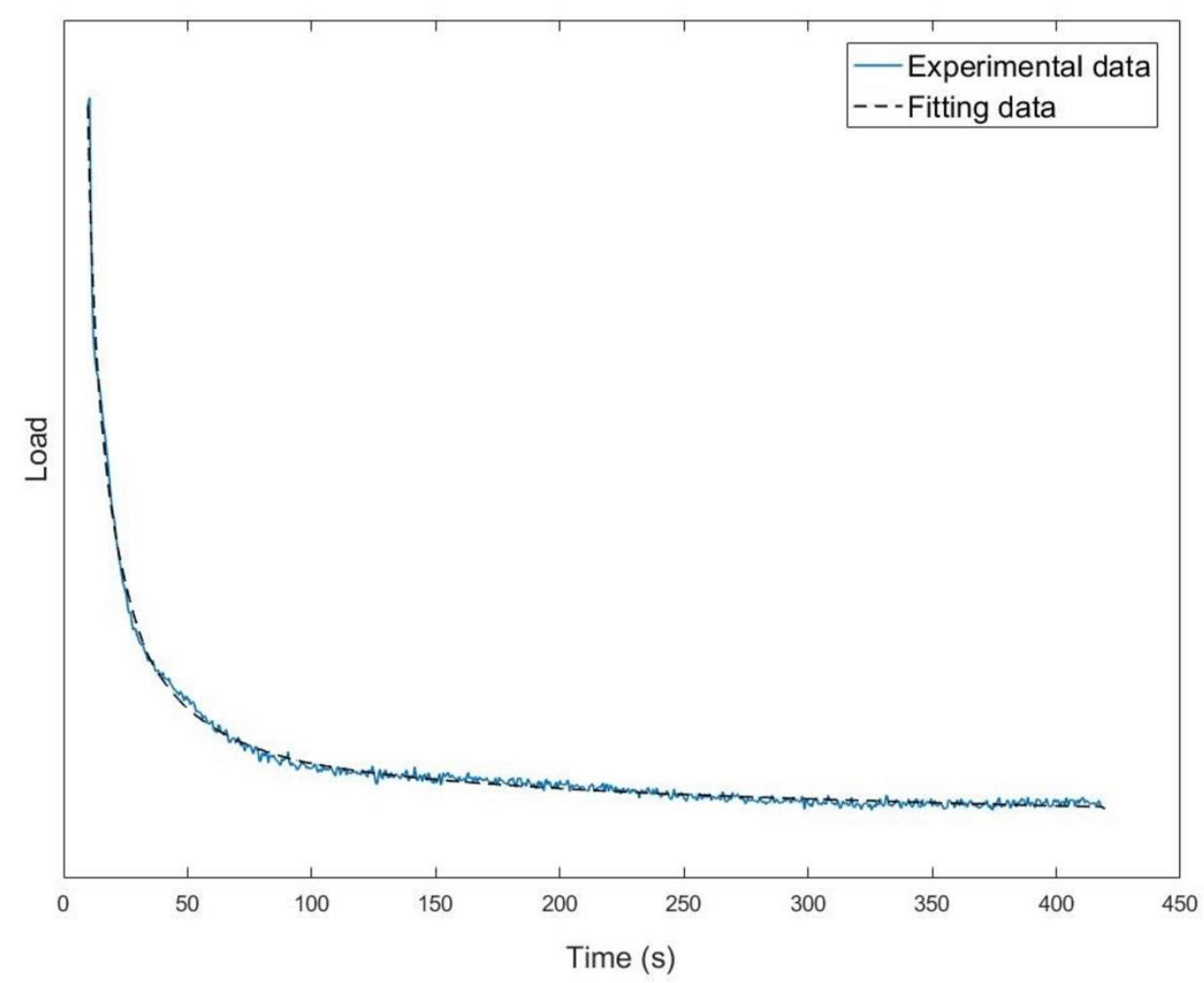

Figure 2: Curves representing the normalized force vs time for the experimental data and viscoelastic model with four branches.

2.3 Calcified cartilage and cortical plate mechanical properties 
For each sample $(n=12)$, sagittal sections in the centre of the MFC that were $3 \mathrm{~mm}$ thick were prepared as follow. The samples were ground on sand paper (\#1200) to obtain planoparallel surfaces then polished using a $1-\mu \mathrm{m}$ diamond powder and rinsed in an ultrasonication bath. Images taken under an optical microscope were assembled to visualize the entire surface of the MFC (Figure 1-C).

Nanoindentation tests were performed following published protocol (35) using a commercial nanoindenter (Agilent Nanoindenter G200, ScienTec, Les Ulis, France) to assess tissue mechanical properties (34). Fused silica was used for calibration of the Berkovich diamond tip contact surface. The location of each indent was set manually in line with a regular spacing of approximately $100 \mu \mathrm{m}$, in the area under the hyaline cartilage previously measured. Eleven points were defined in the ZOI of the condyles for the Ct.Pt and the CC of each sample. To ensure that the points fell in the $\mathrm{CC}$, they were placed approximately $30 \mu \mathrm{m}$ away from the tidemark.

A constant strain rate of $0.05 \mathrm{~s}^{-1}$ and a maximum depth of $2000 \mathrm{~nm}$ were imposed. The Continuous Stiffness Measurement (CSM) method allowed for a determination of Young's modulus as a function of the displacement into the surface. The Oliver and Pharr's method (36) was used for this study. The material was assumed to be linear, elastic and isotropic (37). The Poisson ratio was assumed to be 0.3 for both the Ct.Pt and the CC (38). Young's modulus for each indentation point was averaged on the plateau between 600 and $800 \mathrm{~nm}$.

\subsection{Multiphoton imaging}

Biphotonic confocal microscopy (BCM) was used to investigate the microstructure of the $\mathrm{CC}$ and the Ct.Pt. Second harmonic generation (SHG) from collagen and autofluorescence from elastin fibres were excited by a wavelength of $850 \mathrm{~nm}$, as previously published (39). Lights were collected on two channel-specific band-pass filters of 400-490 and 500-550 nm, 
respectively. A 25x, 1.1 numerical aperture, water immersion objective (CFI Apo LWD $25 \mathrm{XW}$, Nikon) was used. The image field of view was $1024 \times 1024$ pixels with a resolution of $0.5 \mu \mathrm{m}$. Stacks of $2 \mathrm{D}$ images were recorded in the ZOI with a scan speed of 0.125 frame/sec and an average of 2 scans per image every $1 \mu \mathrm{m}$ from 0 to $20 \mu \mathrm{m}$ in depth. For each sample the tortuosity of the cement line was measured using imageJ software (plugin Skeletonize 2D/3D) on the image at $5 \mu \mathrm{m}$ in depth. Tortuosity was defined as the ratio of the meandering line length to the straight line between the end points (40).

\subsection{Statistical analysis}

Statistical analysis was performed using R software (R Foundation for Statistical Computing, Vienna, Austria). Mann-Whitney tests were used to assess the significant differences between mechanical properties (of HC, CC and Ct.Pt) and tortuosity of the control ( $\mathrm{n}=6)$ and ACLT $(n=6)$ groups. Wilcoxon rank-sum tests were performed to evaluate the differences between the Ct.Pt and the CC. The level of significance for all analyses corresponded to an $\alpha$-risk of $5 \%$, and the analysis unit was an animal.

\section{Results}

\subsection{Macroscopic PTOA grading}

The macroscopic degradations of cartilage and osteophyte formation were scored for the control and ACLT groups (table 1). All of the control rabbits displayed rare cartilage alterations and minute osteophytes. The average grade of femoral cartilage degradation was $0.17 \pm 0.41$ on the medial compartment and $0 \pm 0$ in the lateral part. No osteophytes were found on the femoral condyles or on the trochlea. 
Table 1: Grading score of OA lesion, for the control (CTRL) and ACLT groups. ${ }^{*}$ : p-value $<0.05$ between the control and ACLT groups.

\begin{tabular}{|c|c|c|c|c|c|c|}
\hline \multirow[b]{3}{*}{ Group } & \multirow{2}{*}{\multicolumn{2}{|c|}{ Femoral condyle cartilage }} & \multicolumn{4}{|c|}{ Osteophytes } \\
\hline & & & \multicolumn{2}{|c|}{ trochlea } & \multicolumn{2}{|c|}{ femoral condyles } \\
\hline & Medial & Lateral & Medial & Lateral & Medial & Lateral \\
\hline \multirow{6}{*}{ CTRL } & 0 & 0 & 0 & 0 & 0 & 0 \\
\hline & 1 & 0 & 0 & 0 & 0 & 0 \\
\hline & 0 & 0 & 0 & 0 & 0 & 0 \\
\hline & 0 & 0 & 0 & 0 & 0 & 0 \\
\hline & 0 & 0 & 0 & 0 & 0 & 0 \\
\hline & $\underline{0}$ & 0 & $\underline{0}$ & 0 & 0 & $\underline{0}$ \\
\hline $\begin{array}{l}\text { Mean } \\
\text { CTRL } \pm S D\end{array}$ & $0,17 \pm 0.37$ & $0 \pm 0$ & $0 \pm 0$ & $0 \pm 0$ & $0 \pm 0$ & $0 \pm 0$ \\
\hline \multirow{6}{*}{ ACLT } & 1 & 2 & 3 & 2 & 1 & 0 \\
\hline & 1 & 0 & 2 & 0 & 0 & 0 \\
\hline & 1 & 0 & 2 & 0 & 2 & 0 \\
\hline & 1 & 0 & 3 & 0 & 1 & 1 \\
\hline & 2 & 1 & 1 & 0 & 0 & 0 \\
\hline & 1 & 3 & 2 & 1 & 1 & 1 \\
\hline $\begin{array}{l}\text { Mean } \\
\text { ACLT } \pm S D\end{array}$ & $1,17 \pm 0.37 *$ & $1 \pm 1.15$ & $2,17 \pm 0.69 *$ & $0,5 \pm 0.76$ & $0,83 \pm * 0,69$ & $0,33 \pm 0.47$ \\
\hline
\end{tabular}

All of the rabbits from the ACLT group exhibited fibrillation or erosion of femoral cartilage, as well as osteophyte formation, especially in the femoral trochlea. The scores in the medial compartment were significantly higher for the ACLT group than for the control group with a mean value of $1.2 \pm 0.41$ ( $\mathrm{p}$-value $=0.008$ ) for the femoral cartilage degradation as well as $0.83 \pm 0.75$ (p-value $=0.03$ ) and $2.2 \pm 0.84$ (p-value=0.003) for the osteophyte formation on the femoral condyles and on the trochlea, respectively. Complete cartilage erosion was not observed in any rabbit, which indicated an early stage of OA development (41).

\subsection{Hyaline cartilage mechanical properties}

Mechanical properties of the hyaline cartilage obtained by indentation are displayed in Figure 3 and table 2. Instantaneous modulus was not significantly affected by OA (figure 3-A) whereas the equilibrium modulus (figure 3 -B) decreased significantly from $1.47 \pm 0.35 \mathrm{MPa}$ in the control group to $0.85 \pm 0.29 \mathrm{MPa}$ in the ACLT group (p-value=0.02). The elastic fraction 
(figure 3-C), defined as the ratio of $\mathrm{E}_{\mathrm{eq}}$ and $\mathrm{E}_{\mathrm{ins}}$, was also significantly reduced in the ACLT group $(0.30 \pm 0.03)$ compared to the control group $(0.25 \pm 0.03$, $\mathrm{p}$-value $=0.005)$.

Table 2: Comparison of the instantaneous modulus, equilibrium modulus and elastic fraction of the hyaline cartilage between control (CTRL) and ACLT groups. *: p-value $<0.05$ between control (CTRL) and ACLT groups.

\begin{tabular}{|c|c|c|c|c|c|c|}
\hline Groups & & CTRL & & & ACLT & \\
\hline $\begin{array}{l}\text { Mechanical } \\
\text { property }\end{array}$ & $\mathrm{E}_{\text {ins }}$ & $E_{e q}$ & $f$ & $E_{\text {ins }}$ & $E_{e q}$ & $f$ \\
\hline Moduli & 4.75 & 1.91 & 0.40 & 5.15 & 1.05 & 0.20 \\
\hline (MPa) & 3.45 & 1.29 & 0.38 & 4.07 & 1.11 & 0.27 \\
\hline & 5.08 & 1.98 & 0.39 & 1.34 & 0.33 & 0.25 \\
\hline & 3.39 & 1.21 & 0.36 & 2.55 & 0.68 & 0.27 \\
\hline & 3.12 & 1.39 & 0.45 & 4.07 & 1.16 & 0.28 \\
\hline & 2.93 & 1.05 & 0.36 & 3.04 & 0.75 & 0.25 \\
\hline mean $\pm S D$ & $3.79 \pm 0.82$ & $1.47 \pm 0.35$ & $0.39 \pm 0.03$ & $3.37 \pm 1.23$ & $0.85 \pm 0.29 *$ & $0.25 \pm 0.03 *$ \\
\hline
\end{tabular}
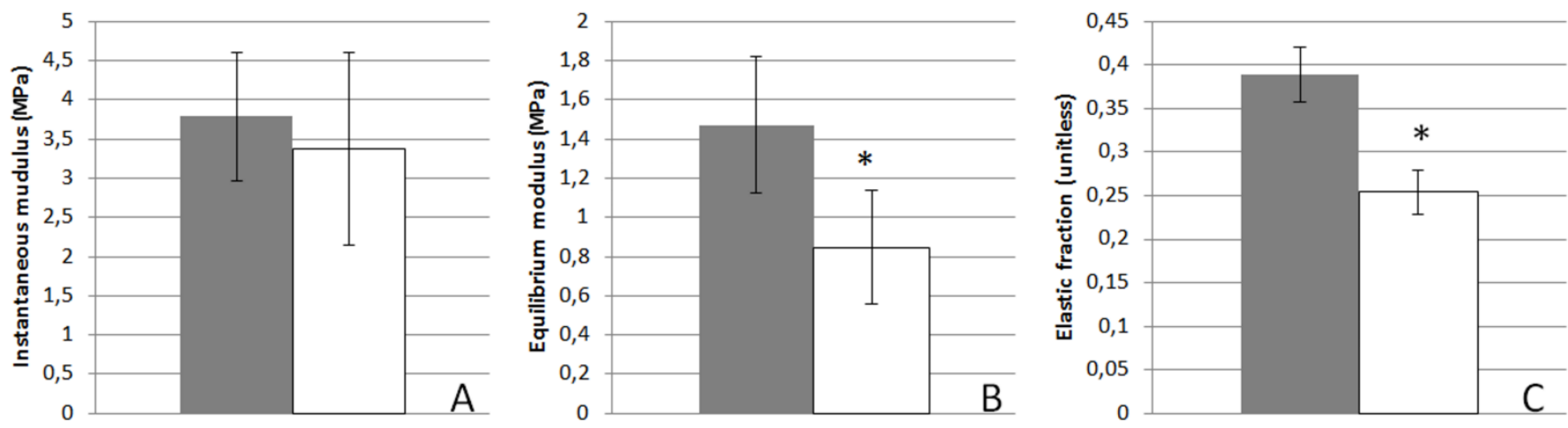

$\because \mathrm{CTRL}$

$\square$ ACLT

Figure 3: Hyaline cartilage mean values of the instantaneous modulus (A), equilibrium modulus (B) and the elastic fraction (C) for the control (CTRL) and ACLT groups. Bars show the mean values and the error bars correspond to standard deviations. *: p-value $<0.05$ between the control and ACLT groups.

3.3 Calcified cartilage and cortical plate mechanical properties

Average values of the elastic modulus, which was obtained using nanoindentation technique of both the Ct.Pt and the CC, decreased significantly (figure 4-A) for the ACLT group compared to the control group. The average value of the elastic modulus displayed a reduction of $16 \%$ in the Ct.Pt (from $17.31 \pm 1.27 \mathrm{GPa}$ in the control group to $14.49 \pm 1.43 \mathrm{GPa}$ 
in the ACLT group, p-value=0.02) and $37 \%$ in the CC (from $15.62 \pm 0.90 \mathrm{GPa}$ in the control group to $9.85 \pm 1.30 \mathrm{GPa}$ in the ACLT group, $\mathrm{p}$-value=0.002). The average values of the elastic moduli of the Ct.Pt was significantly higher than in the CC from the ACLT group (pvalue $=0.03$ ), but not in the control group. Similarly, average hardness (figure 4-B) decreased significantly in the Ct.Pt (from $0.72 \pm 0.10 \mathrm{GPa}$ in the control group to $0.57 \pm 0.10 \mathrm{GPa}$ in the ACLT group, p-value=0.04) and in the $\mathrm{CC}$ (from $0.70 \pm 0.06 \mathrm{GPa}$ in the control group to $0.39 \pm 0.09 \mathrm{GPa}$ in the ACLT group, $\mathrm{p}$-value $=0.002$ ).
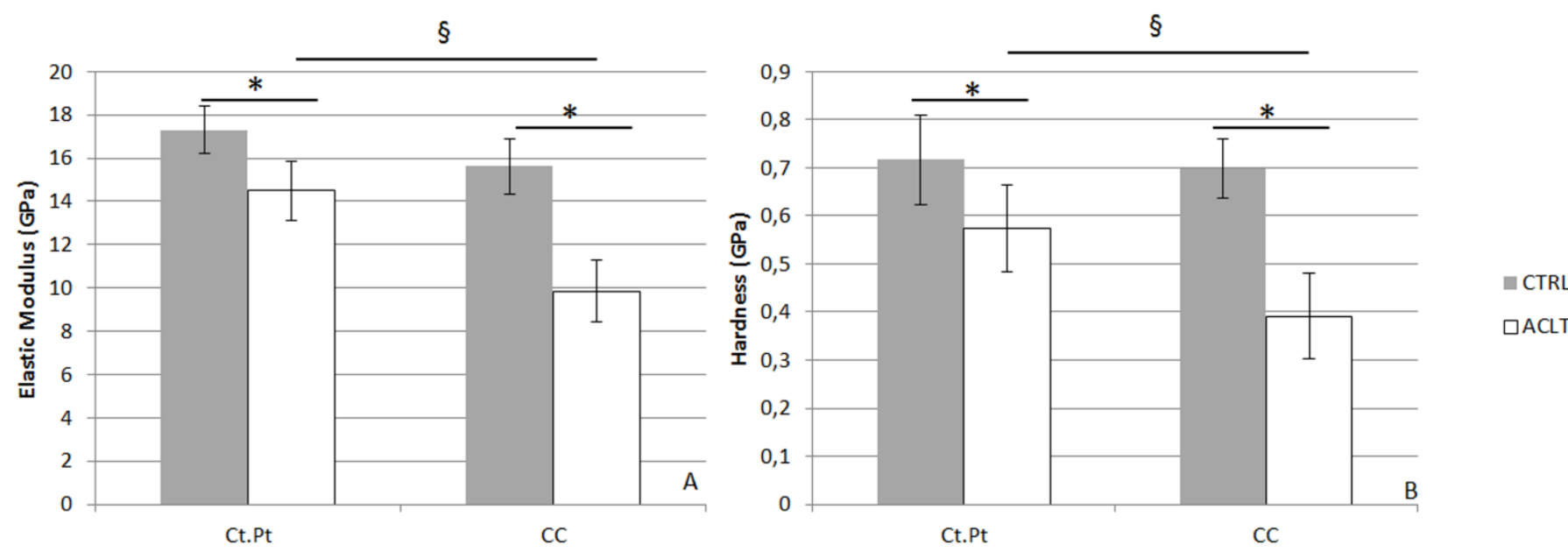

Figure 4: Mean values of the elastic modulus (A) and hardness (B) of the cortical plate (Ct. Pt) and the calcified cartilage (CC) for the control (CTRL) and ACLT groups. Bars show the mean values and the error bars correspond to standard deviations. *: p-value value $<0.05$ between the control and ACLT groups, $\S$ : $p$-value $<0.05$ between cortical plate (Ct.PI) and calcified cartilage (CC).

\subsection{Collagen organization}

Ct.Pt and $\mathrm{CC}$ were clearly identifiable in both groups due to their respective and distinct collagen fibre organization. In the Ct.Pt, no privileged orientation was observed, whereas in the $\mathrm{CC}$, the fibres were parallel between each other and perpendicular to the tidemark. 

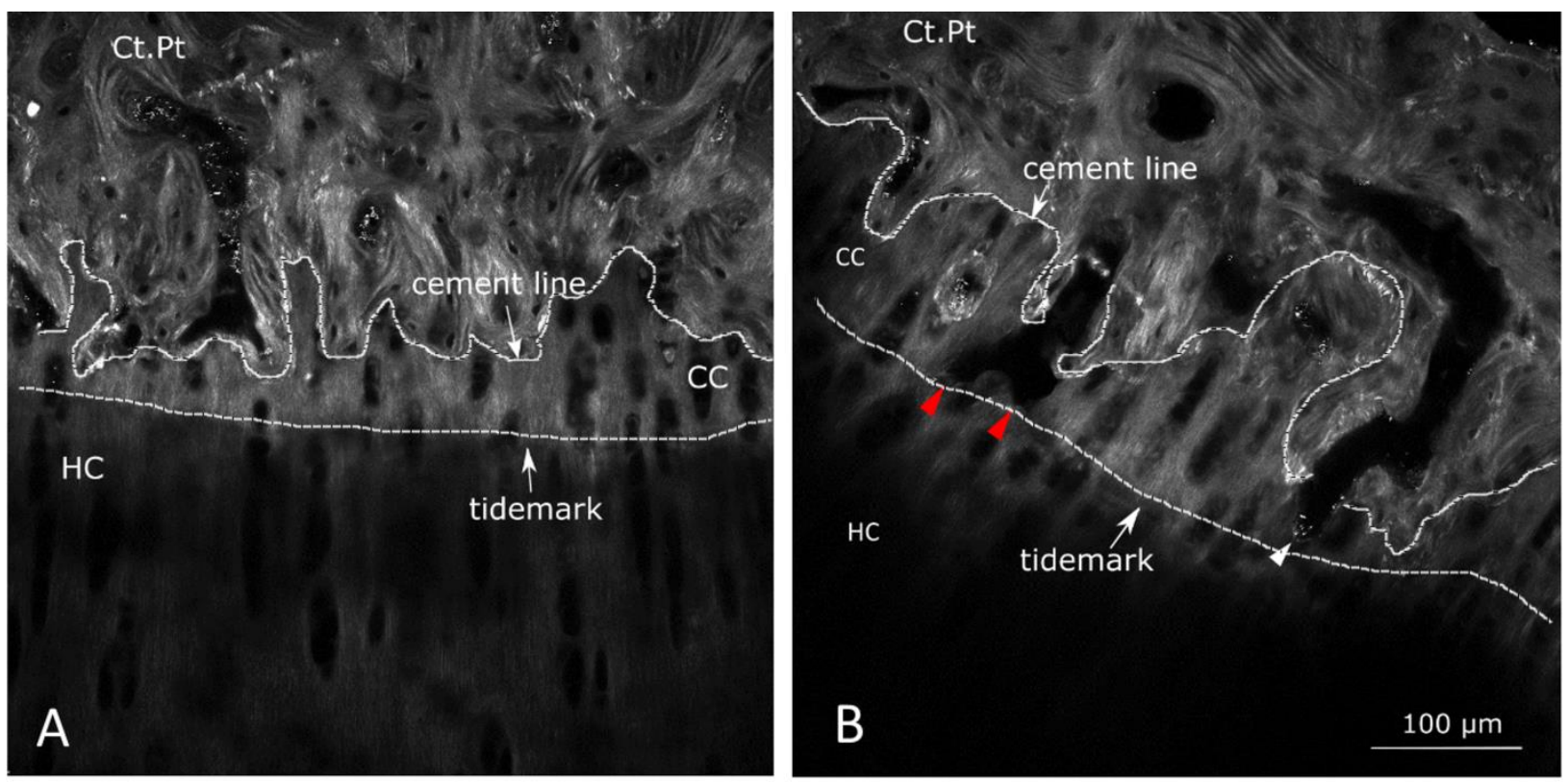

Figure 2: Second Harmonic Generation (SHG) images of the osteochondral junction highlighting the collagen matrix at a $5 \mu \mathrm{m}$ depth under the surface. A: Typical image of a sample in the control group. B: Typical image of a sample in the ACLT group. Multiple channels reaching the tidemark (red arrowheads) and open channels crossing the cement line (white arrow head) were clearly observed in the ACLT group. Ct.Pt: Cortical Plate, CC: Calcified Cartilage, HC: Hyaline Cartilage.

The osteochondral junction displayed a different structural organization in the ZOI of the ACLT group than in the ZOI of the control group (Fig 5). In particular, the cement line presented stronger invaginations, which was measured by its tortuosity (table 3 ). The mean value of the tortuosity was significantly higher in the ACLT group $(3.37 \pm 0.89)$ compared to the control group $(2.19 \pm 0.41, \mathrm{p}$-value $=0.04)$. This led to strong thickness variation of the CC from 0 to $300 \mu \mathrm{m}$ in the ACLT group. Moreover, channels from the Ct.Pt crossing the cement line and reaching the $\mathrm{CC}$ were more present in the ACLT group than in the control group, and several channels even reached the tidemark (figure 5-B).

Table 3: Comparison of the tortuosity index between both control (CTRL) and ACLT groups. *: p-value $<0.05$ between control and ACLT groups.

\begin{tabular}{|c|r|r|}
\hline Groups & CTRL $(\mathbf{n}=6)$ & ACLT $(\mathbf{n}=6)$ \\
\hline Tortuosity & 1.72 & 4.82 \\
\hline
\end{tabular}




\begin{tabular}{|c|r|r|}
\hline \multirow{3}{*}{$\begin{array}{c}\text { index } \\
\text { (unitless) }\end{array}$} & 1.99 & 2.73 \\
\cline { 2 - 3 } & 1.78 & 2.50 \\
\cline { 2 - 3 } & 2.67 & 2.42 \\
\cline { 2 - 3 } & 2.21 & 3.73 \\
\cline { 2 - 3 } & 2.78 & 4.01 \\
\hline mean \pm SD & $\mathbf{2 . 1 9} \pm \mathbf{0 . 4 1}$ & $\mathbf{3 . 3 7} \pm \mathbf{0 , 8 9}$ \\
\hline
\end{tabular}

\section{Discussion}

In the present study, the mechanical and structural properties of the hyaline cartilage, calcified cartilage and cortical plate were compared between healthy (control group) and PTOA (ACLT group) rabbit knees through mechanical testing (nanoindentation) and imaging methods (biphoton confocal microscopy and environmental scanning electron microscopy). These techniques provided complementary data to investigate mechanical changes of the bone-cartilage unit in parallel with its morphological modifications 6 weeks post-ACLT. Macroscopic grading of the joints demonstrated that all rabbits in the ACLT group developed early PTOA. As several studies have reported OA changes in the contralateral limb of operated animals $(33,42)$, a non-operated (healthy) control group was used. Experiments focused on the medial femoral condyles because macroscopically this compartment was significantly the most affected, which was consistent with other studies of this PTOA model $(17,28,43)$.

To the best of our knowledge, our study is the first to simultaneously investigate mechanical alterations of the soft and hard tissues composing the bone-cartilage unit in a rabbit model of PTOA. The results indicate an overall decrease of the mechanical properties of the hyaline cartilage, calcified cartilage and cortical plate in early OA. Morphological modifications of the osteochondral junction accompanied these mechanical alterations. 
Viscoelastic properties of the hyaline cartilage have been assessed with a model previously published by Levillain et al (33). Three parameters have been compared between control and ACLT groups: the instantaneous modulus, the equilibrium modulus and the elastic fraction. The elastic fraction describes the relative contributions of the elastic and viscous behaviour of the tissue: $\mathrm{f}=1$ indicates a perfectly elastic material whereas $\mathrm{f}=0$ indicates a perfectly viscous material. The elastic fraction has also been correlated with the phase angle assessed by dynamic mechanical testing (44). Hyaline cartilage is a soft biological tissue whose behaviour is time dependent; therefore it is considered as a viscous-elastic material (45). In this study, a viscoelastic model has been chosen to compare control and ACLT groups because it provided satisfying fits of the experimental data and permitted comparisons with previously published studies $(44,46)$. This is a phenomenological model which considers hyaline cartilage as a homogeneous solid and opposite to the biphasic poroelastic model, does not distinguish the solid matrix from the interstitial fluid. Two mechanisms are associated with strain energy dissipation in hyaline cartilage: fluid flow dependent behaviour caused by the circulation of the interstitial fluid and flow independent viscous behaviour of the solid matrix $(46,47)$. A more complex biphasic poroelastic model (as used in $(48,49)$ ) would better match the microscopic behaviour of articular cartilage and would allow to account for the changes in permeability that occur in OA (47). It will be used in future work to provide complementary information. In this work, the mean value of the elastic moduli were within the range of values found in the literature $(43,50,51)$, though direct comparison is not pertinent because of the use of different testing protocol. However, the dramatic decrease of $42 \%$ of the equilibrium modulus of hyaline cartilage in the ACLT group compared with the control group is in accordance with the results of Desrochers et al. (44) who used a canine model of ACLT. Similarly, they found a change toward a more viscous behaviour of the hyaline cartilage, as indicated by the reduction of the elastic fraction. A recent study on 
ACLT rabbits has also shown a decrease in the dynamic modulus of the hyaline cartilage in the medial femoral condyle, but they did not study the elastic fraction (43). This decrease of the hyaline cartilage stiffness following ACLT is not specific of animal models. Vasara et al. (52) performed arthroscopic indentation of the hyaline cartilage of patients undergoing surgery after anterior cruciate ligament rupture. They observed a decrease of the stiffness even when no cartilage damages were yet visible by arthroscopy. However, no information is available on the viscoelastic properties of cartilage in ACL deficient human knees. Yet, this might be an important income as viscoelastic properties of the cartilage contribute to the regulations of the chondrocytes' functions (53).

Nanoindentation of the mineralised tissues of the bone-cartilage unit (cortical plate and calcified cartilage) in the weight-bearing area provided information on the elastic modulus and on the contact hardness. Both cortical plate and calcified cartilage exhibited a decrease in the mechanical properties. When studying the effects of OA in the cortical plate of human femoral heads, Ferguson et al. (11) did not find any difference in elastic modulus between end-stage OA and normal post mortem samples. In contrast, Li and Aspden (20) demonstrated that the mineral content as well as the elastic modulus in the Cortical plate of femoral heads with end-stage OA were significantly reduced compared to healthy tissues. Interestingly, Hargrave-Thomas et al. (54) showed that, in the cortical plate of bovine patellae, the elastic modulus was decreased in early OA samples but then was back to normal in samples with a higher stage of OA. Regarding the calcified cartilage, they showed that elastic modulus tended to diminish but no significant differences were found between the samples with and without OA degradations.

In the control group, calcified cartilage and cortical plate have close values. In the ACLT group, elastic moduli of the tissues decreased by $37 \%$ in the calcified cartilage and $16 \%$ in the cortical plate. As a consequence, the mean elastic modulus of the calcified cartilage is 
significantly lower than that of the cortical plate in this group. Similarly, Hargrave-Thomas et al. (54) found higher elastic modulus values of the cortical plate compared to calcified cartilage in early OA.

One limitation of our study is that the indentation of mineralised tissues was not performed in the physiological loading direction unlike it was in hyaline cartilage. As one of our major aims was to compare the evolution of mechanical properties of both calcified tissues simultaneously, we chose to perform indentation in the same conditions for these two tissues. In a technical point of view and due to the complexity of condyle geometry, it is particularly challenging to measure mechanical properties of calcified tissues in the physiological loading direction. As these tissues exhibit an anisotropic behaviour, elastic moduli in the other direction should have greater absolute values.

Mechanical alterations in the ACLT group were accompanied by morphological changes in the bone-cartilage unit, especially in the osteochondral junction. The irregularity of the cement line, as quantified by a tortuosity index, was accentuated in the ACLT group compared with the control group. An increase in the cement line irregularity in OA were also reported by Thambyah et al. in bovine $(9,55,56)$, as well as in human tissues $(57)$, that ranged from healthy to mild OA. Interestingly, the irregularity of the cement line has been described as a consequence of bone remodelling of the osteochondral junction (2) leading to bone invagination inside calcified cartilage. In the ACLT group, BCM images also revealed an increased number of channels in the calcified cartilage. Some PTOA samples even displayed obvious channels reaching the hyaline cartilage. In particular, a large number of open and closed channels were clearly noticed in ACLT osteochondral junction. According to Clark (58), these channels correspond to Haversian channels that revealed osteochondral ossification meaning that Cortical plate progressed into the calcified cartilage. Aho et al. (59) suggested taking into account subchondral bone for OA grading. Since cement line tortuosity 
appears to be a good indicator of calcified cartilage quality, it should also be part of OA assessment.

Similar to humans suffering from ACL rupture $(31,32,60-62)$, ACL transection in rabbits alters their gait and induces abnormal joint kinematics. According to Mansour et al (28), changes in adduction and external rotation are clearly more pronounced following ACLT in rabbits. Consequently, in the ACLT model, weight-bearing stress was modified in the ZOI. This disruption of the stress pattern induced alterations of the tissues composing the bonecartilage unit. In the ACLT group, our results showed that the most affected tissue in term of mechanical property is the closest to the articular surface. Indeed, hyaline cartilage was the most affected, followed by the calcified cartilage and then the cortical plate in terms of mechanical properties with a decrease of ACLT values compared to control values of $42.4 \%$, $37.0 \%$ and $16.3 \%$ respectively. The relative reductions of the mean elastic modulus of the hyaline cartilage and the calcified cartilage were in the same order of magnitude whereas it was clearly less pronounced in the cortical plate. As a consequence, the stiffness gradient between cartilage and bone was more pronounced in ACLT group compared to healthy group. Radin and Rose (63) hypothesised that initiation and progression of OA was triggered by a stiffness gradient between articular cartilage (hyaline cartilage and calcified cartilage) and the underlying subchondral bone. The present study highlighted that such a stiffness gradient does exist in rabbit PTOA samples at 6 weeks post-ACLT. However, this gradient is essentially due to a strong decrease in the articular cartilage (composed of hyaline cartilage and calcified cartilage) mechanical properties instead of an increase in the elastic modulus of the bone as suggested in Radin and Rose (63). The steeper decrease in the mechanical properties of the articular cartilage, superficial, compared with the deeper subchondral bone might mean that PTOA alterations following ACLT are first initiated in the hyaline cartilage and then propagate deeply towards the bone. 
The results of indentation experiments are in agreement with findings of cartilage softening in clinical studies on human patients with ACL knee deficiency (64) indicating that ACLT in rabbit is a relevant PTOA model. Nonetheless even if small animal models provide useful information about the development of PTOA, they cannot perfectly mimic physiological changes occurring in human patient. However the rupture of the anterior cruciate ligament in rabbits is a well-established model $(17,21,25-27)$ that results in joint instability (28) as it has been observed in human patients suffering from ACL deficiency $(31,32)$. These instabilities are assumed to increase the risk of developing OA in human patients $(32,52)$, and PTOA patients represent a large part of the overall OA population (65). Nevertheless, there is not just one type of OA but rather a wide spectrum of degenerative changes with different aetiologies (66) that are studied in vivo with other animal models $(24,67)$. The rabbit model of PTOA used in this study provides insights only on the early phase of OA imputable to joint instabilities.

In conclusion, in the ACLT rabbit model, early PTOA induced adaptions of the bonecartilage unit in terms of mechanical and morphological properties in the MFC. A strong decrease in the stiffness of the hyaline cartilage, calcified cartilage and cortical plate was observed, hyaline and calcified cartilage being the most affected. These results provide a better understanding of the alteration of the load distribution occurring in early OA. Further studies will aim to evaluate the effect of therapy on their ability to maintain bone-cartilage unit functional properties. 


\section{References}

1. Lories RJ, Luyten FP. The bone-cartilage unit in osteoarthritis. Nat Rev Rheumatol. 2011 Jan;7(1):43.

2. Ferguson VL, Paietta RC. The bone-cartilage interface. In 2013. p. 91-118.

3. Mansfield JC, Peter Winlove C. A multi-modal multiphoton investigation of microstructure in the deep zone and calcified cartilage. J Anat. 2012;220(4):405-16.

4. Burr DB, Gallant MA. Bone remodelling in osteoarthritis. Nat Rev Rheumatol. 2012;8(11):66573.

5. Felson DT. Osteoarthritis as a disease of mechanics. Osteoarthritis Cartilage. 2013;21(1):10-5.

6. Burr DB. Anatomy and physiology of the mineralized tissues: Role in the pathogenesis of osteoarthrosis. Osteoarthritis Cartilage. 2004;12, Supplement:20-30.

7. Oegema TR, Carpenter RJ, Hofmeister F, Thompson RC. The interaction of the zone of calcified cartilage and subchondral bone in osteoarthritis. Microsc Res Tech. 1997;37(4):324332.

8. Norrdin RW, Kawcak CE, Capwell BA, McIlwraith CW. Calcified cartilage morphometry and its relation to subchondral bone remodeling in equine arthrosis. Bone. 1999;24(2):109-14.

9. Thambyah A, Broom N. On new bone formation in the pre-osteoarthritic joint. Osteoarthritis Cartilage. 2009 Apr 1;17(4):456-63.

10. Arkill KP, Winlove CP. Solute transport in the deep and calcified zones of articular cartilage. Osteoarthritis Cartilage. 2008;16(6):708-14.

11. Ferguson VL, Bushby AJ, Boyde A. Nanomechanical properties and mineral concentration in articular calcified cartilage and subchondral bone. J Anat. 2003;203(2):191-202.

12. Richard F, Villars M, Thibaud S. Viscoelastic modeling and quantitative experimental characterization of normal and osteoarthritic human articular cartilage using indentation. J Mech Behav Biomed Mater. 2013 Aug 1;24(Supplement C):41-52.

13. Podsiadlo P, Dahl L, Englund M, Lohmander LS, Stachowiak GW. Differences in trabecular bone texture between knees with and without radiographic osteoarthritis detected by fractal methods. Osteoarthritis Cartilage. 2008 Mar 1;16(3):323-9.

14. Eckstein F, Guermazi A, Roemer FW. Quantitative MR Imaging of Cartilage and Trabecular Bone in Osteoarthritis. Radiol Clin North Am. 2009 Jul 1;47(4):655-73.

15. Tomanik M, Nikodem A, Filipiak J. Microhardness of human cancellous bone tissue in progressive hip osteoarthritis. J Mech Behav Biomed Mater. 2016 Dec 1;64(Supplement C):8693.

16. Bouchgua M, Alexander K, Norman Carmel E, d'Anjou MA, Beauchamp G, Richard H, et al. Use of routine clinical multimodality imaging in a rabbit model of osteoarthritis - part II: bone mineral density assessment. Osteoarthritis Cartilage. 2009;17(2):197-204.

17. Florea C, Malo MKH, Rautiainen J, Mäkelä JTA, Fick JM, Nieminen MT, et al. Alterations in subchondral bone plate, trabecular bone and articular cartilage properties of rabbit femoral 
condyles at 4 weeks after anterior cruciate ligament transection. Osteoarthritis Cartilage. 2015;23(3):414-22.

18. Kuroki K, Cook CR, Cook JL. Subchondral bone changes in three different canine models of osteoarthritis. Osteoarthritis Cartilage. 2011;19(9):1142-9.

19. Rieger R, Boulocher C, Kaderli S, Hoc T. Chitosan in viscosupplementation: in vivo effect on rabbit subchondral bone. BMC Musculoskelet Disord. 2017 Aug 15;18:350.

20. Li B, Aspden RM. Mechanical and material properties of the subchondral bone plate from the femoral head of patients with osteoarthritis or osteoporosis. Ann Rheum Dis. 1997;56(4):24754.

21. Saito M, Sasho T, Yamaguchi S, Ikegawa N, Akagi R, Muramatsu Y, et al. Angiogenic activity of subchondral bone during the progression of osteoarthritis in a rabbit anterior cruciate ligament transection model. Osteoarthritis Cartilage. 2012;20(12):1574-82.

22. Suri S, Gill SE, Massena de Camin S, McWilliams DF, Wilson D, Walsh DA. Neurovascular invasion at the osteochondral junction and in osteophytes in osteoarthritis. Ann Rheum Dis. 2007;66(11):1423-8.

23. Lampropoulou-Adamidou K, Lelovas P, Karadimas EV, Liakou C, Triantafillopoulos IK, Dontas I, et al. Useful animal models for the research of osteoarthritis. Eur J Orthop Surg Traumatol. 2014;24(3):263-71.

24. Bendele A. Animal models of osteoarthritis. J Musculoskelet Neuronal Interact. 2001;1(4):363376.

25. Laverty S, Girard CA, Williams JM, Hunziker EB, Pritzker KPH. The OARSI histopathology initiative - recommendations for histological assessments of osteoarthritis in the rabbit. Osteoarthritis Cartilage. 2010 Oct 1;18(Supplement 3):S53-65.

26. Batiste DL, Kirkley A, Laverty S, Thain LMF, Spouge AR, Holdsworth DW. Ex-vivo characterization of articular cartilage and bone lesions in a rabbit ACL transection model of osteoarthritis using MRI and micro-CT. Osteoarthritis Cartilage. 2004;(12):986-96.

27. Sah RL, Yang AS, Chen AC, Hant JJ, Halili RB, Yoshioka M, et al. Physical properties of rabbit articular cartilage after transection of the anterior cruciate ligament. J Orthop Res. 1997;15(2):197-203.

28. Mansour JM, Wentorf FA, Degoede KM. In Vivo Kinematics of the Rabbit Knee in Unstable Models of Osteoarthrosis. Ann Biomed Eng. 1997;26(3):353-360.

29. Kuyinu EL, Narayanan G, Nair LS, Laurencin CT. Animal models of osteoarthritis: classification, update, and measurement of outcomes. J Orthop Surg. 2016 Feb 2;11:19.

30. McCoy AM. Animal Models of Osteoarthritis: Comparisons and Key Considerations. Vet Pathol. 2015 Sep 1;52(5):803-18.

31. Bonnin M, Carret JP, Dimnet J, Dejour H. The weight-bearing knee after anterior cruciate ligament rupture. Knee Surg Sports Traumatol Arthrosc. 1996 Mar 1;3(4):245-51.

32. Louboutin H, Debarge R, Richou J, Selmi TAS, Donell ST, Neyret P, et al. Osteoarthritis in patients with anterior cruciate ligament rupture: A review of risk factors. The Knee. 2009 Aug 1;16(4):239-44. 
33. Levillain A, Magoariec H, Boulocher C, Decambron A, Viateau V, Hoc T. Viscoelastic properties of rabbit osteoarthritic menisci: A correlation with matrix alterations. J Mech Behav Biomed Mater. 2017 Jan 1;65(Supplement C):1-10.

34. Vaseenon T, Tochigi Y, Heiner AD, Goetz JE, Baer TE, Fredericks DC, et al. Organ-level histological and biomechanical responses from localized osteoarticular injury in the rabbit knee. J Orthop Res. 2011;29(3):340-346.

35. Imbert L, Aurégan J-C, Pernelle K, Hoc T. Mechanical and mineral properties of osteogenesis imperfecta human bones at the tissue level. Bone. 2014 Aug;65:18-24.

36. Oliver WC, Pharr GM. Improved technique for determining hardness and elastic modulus using load and displacement sensing indentation experiments. J Mater Res. 1992;7(6):1564-80.

37. Zysset PK, Guo XE, Hoffler CE, Moore KE, Goldstein SA. Elastic modulus and hardness of cortical and trabecular bone lamellae measured by nanoindentation in the human femur. $\mathrm{J}$ Biomech. 1999;32(10):1005-12.

38. Stender ME, Regueiro RA, Ferguson VL. A poroelastic finite element model of the bonecartilage unit to determine the effects of changes in permeability with osteoarthritis. Comput Methods Biomech Biomed Engin. 2017 Feb 17;20(3):319-31.

39. Levillain A, Orhant M, Turquier F, Hoc T. Contribution of collagen and elastin fibers to the mechanical behavior of an abdominal connective tissue. J Mech Behav Biomed Mater. 2016 Aug 1;61(Supplement C):308-17.

40. Heneghan C, Flynn J, O'Keefe M, Cahill M. Characterization of changes in blood vessel width and tortuosity in retinopathy of prematurity using image analysis. Med Image Anal. 2002;6(4):407-429.

41. Hargrave-Thomas E, van Sloun F, Dickinson M, Broom N, Thambyah A. Multi-scalar mechanical testing of the calcified cartilage and subchondral bone comparing healthy vs early degenerative states. Osteoarthritis Cartilage. 2015;23(10):1755-62.

42. Bray RC, Shrive NG, Frank CB, Chimich DD. The early effects of joint immobilization on medial collateral ligament healing in an ACL-deficient knee: A gross anatomic and biomechanical investigation in the adult rabbit model. J Orthop Res. 1992;10(2):157-166.

43. Mäkelä JTA, Rezaeian ZS, Mikkonen S, Madden R, Han S-K, Jurvelin JS, et al. Site-dependent changes in structure and function of lapine articular cartilage 4 weeks after anterior cruciate ligament transection. Osteoarthritis Cartilage. 2014 Jun 1;22(6):869-78.

44. Desrochers J, Amrein MW, Matyas JR. Viscoelasticity of the articular cartilage surface in early osteoarthritis. Osteoarthritis Cartilage. 2012 May 1;20(5):413-21.

45. Oyen ML. Nanoindentation of Biological and Biomimetic Materials. Exp Tech. 2013 Jan 1;37(1):73-87.

46. Setton LA, Mow VC, Howell DS. Mechanical behavior of articular cartilage in shear is altered by transection of the anterior cruciate ligament. J Orthop Res. 1995 Jul 1;13(4):473-82.

47. Setton LA, Mow VC, Müller FJ, Pita JC, Howell DS. Mechanical Properties of Canine Articular Cartilage Are Significantly Altered Following Transection of the Anterior Cruciate Ligament. J Orthop Res. 1994 Jul 1;12(4):451-63. 
48. Berteau J-P, Oyen M, Shefelbine SJ. Permeability and shear modulus of articular cartilage in growing mice. Biomech Model Mechanobiol. 2016 Feb 1;15(1):205-12.

49. Mow VC, Gibbs MC, Lai WM, Zhu WB, Athanasiou KA. Biphasic indentation of articular cartilage-II. A numerical algorithm and an experimental study. J Biomech. 1989 Jan 1;22(8):853-61.

50. Tomkoria S, Patel RV, Mao JJ. Heterogeneous nanomechanical properties of superficial and zonal regions of articular cartilage of the rabbit proximal radius condyle by atomic force microscopy. Med Eng Phys. 2004 Dec 1;26(10):815-22.

51. Hu K, Radhakrishnan P, Patel RV, Mao JJ. Regional Structural and Viscoelastic Properties of Fibrocartilage upon Dynamic Nanoindentation of the Articular Condyle. J Struct Biol. 2001 Oct 1;136(1):46-52.

52. Vasara AI, Jurvelin JS, Peterson L, Kiviranta I. Arthroscopic Cartilage Indentation and Cartilage Lesions of Anterior Cruciate Ligament-Deficient Knees. Am J Sports Med. 2005 Mar 1;33(3):408-14.

53. Lee H, Gu L, Mooney DJ, Levenston ME, Chaudhuri O. Mechanical confinement regulates cartilage matrix formation by chondrocytes. Nat Mater. 2017 Dec;16(12):1243.

54. Hargrave-Thomas E, van Sloun F, Dickinson M, Broom N, Thambyah A. Multi-scalar mechanical testing of the calcified cartilage and subchondral bone comparing healthy vs early degenerative states. Osteoarthritis Cartilage. 2015 Oct 1;23(10):1755-62.

55. Thambyah A, Broom N. On how degeneration influences load-bearing in the cartilage-bone system: a microstructural and micromechanical study. Osteoarthritis Cartilage.

2007;15(12):1410-23.

56. Thambyah A, Zhao J-Y, Bevill SL, Broom ND. Macro-, micro- and ultrastructural investigation of how degeneration influences the response of cartilage to loading. J Mech Behav Biomed Mater. 2012 Jan 1;5(1):206-15.

57. Hargrave-Thomas EJ, Thambyah A, McGlashan SR, Broom ND. The bovine patella as a model of early osteoarthritis. J Anat. 2013;223(6):651-664.

58. Clark JM. The structure of vascular channels in the subchondral plate. J Anat. 1990;171:105.

59. Aho O-M, Finnilä M, Thevenot J, Saarakkala S, Lehenkari P. Subchondral bone histology and grading in osteoarthritis. PLoS ONE. 2017;12(3).

60. Sommerlath K, Lysholm J, Gillquist J. The long-term course after treatment of acute anterior cruciate ligament ruptures: A 9 to 16 year followup. Am J Sports Med. 1991 Mar 1;19(2):15662.

61. Shirakura K, Terauchi M, Kizuki S, Moro S, Kimura M. The natural history of untreated anterior cruciate tears in recreational athletes. Clin Orthop. 1995 Aug;(317):227-36.

62. Fetto JF, Marshall JL. The natural history and diagnosis of anterior cruciate ligament insufficiency. Clin Orthop. 1980;(147):29-38.

63. Radin EL, Rose RM. Role of Subchondral Bone in the Initiation and Progression of Cartilage Damage. Clin Orthop. 1986 Dec;213:34. 
64. Laasanen MS, TÖyrÄs J, Vasara AI, Hyttinen MM, Saarakkala S, Hirvonen J, et al. Mechanoacoustic Diagnosis Of Cartilage Degeneration And Repair. J Bone Jt Surg-Am Vol. 2003 Jan $1 ; 85: 78-84$.

65. Lohmander LS, Englund PM, Dahl LL, Roos EM. The Long-term Consequence of Anterior Cruciate Ligament and Meniscus Injuries: Osteoarthritis. Am J Sports Med. 2007 Oct $1 ; 35(10): 1756-69$.

66. Cadet C, Maheu E, Breville P, Jeandel C, Le Quintrec J-., Lévy-Reynaud O, et al. Clinical phenotypes: There is not one osteoarthritis but several. Comorbidities. Cah Annee Gerontol. 2015;7(2):52-7.

67. Teeple E, Jay GD, Elsaid KA, Fleming BC. Animal Models of Osteoarthritis: Challenges of Model Selection and Analysis. AAPS J. 2013 Jan 18;15(2):438-46. 